\title{
EL ABP CON EL USO DE LAS TIC EN LA ENSEÑANZA DE LA INTELIGENCIA ARTIFICIAL
}

\author{
Yadisbel Martínez Cañete, Dr. C \\ Universidad de Oriente, Cuba \\ ymartinez@uo.edu.cu
}

\author{
Miriam Roll Hechavarría, Dr. C. \\ Universidad de Oriente, Cuba \\ miriamrh@uo.edu.cu
}

\author{
Lucía Sotomayor Guevara, MSc. \\ Universidad de Oriente, Cuba \\ sotomayor@uo.edu.cu
}

Palabras claves: Aprendizaje Basado en Problemas, Inteligencia Artificial, TIC, Moodle.

Keywords: Problem-based learning, Artificial Intelligence, TIC, Moodle
Recibido: 04 de Septiembre de 2017

Aceptado: 12 de Octubre de 2017

\section{RESUMEN}

La carrera de Ingeniería Informática tiene como objetivo en la formación del profesional, que los estudiantes puedan elaborar sistemas informáticos de aplicación para la gestión y toma de decisiones en organizaciones productivas y de servicio, dentro de sus disciplinas se encuentra la de Inteligencia Artificial, la cual se centra en el estudio de modelos y métodos de representación y solución de problemas empleando técnicas de Inteligencia Artificial, en su desarrollo se abordan temas complejos, que requieren del pensamiento abstracto del estudiante, lo cual tradicionalmente ha generado falta de motivación de los estudiantes, en este sentido, se hace necesario la utilización por el docente de métodos de enseñanza, que favorezca el protagonismo e implicación del estudiante en el aprendizaje aprovechando las bondades de las Tecnologías de la Información y las Comunicaciones, y las preferencias que tienen para su utilización. En este trabajo se tiene en cuenta las posibilidades del método de enseñanza Aprendizaje Basado en Problemas, así como, otras propuestas metodológicas que propician el trabajo en grupo y la comunicación on-line entre los alumnos, utilizando un entorno Moodle 2.0 con herramientas de trabajo grupal y OpenMeetings.

\begin{abstract}
The career of Informatics Engineering aims at the training of professionals, that the students can elaborate computer systems of application for the management and decision making in productive and service organizations, within its disciplines is the one of Artificial Intelligence, which focuses on the study of models and methods of representation and solution of problems using techniques of Artificial Intelligence, complex issues are addressed in its development, which require abstract thinking of the student, which has traditionally generated a lack of student motivation, in this sense, It is necessary the use by the teacher of teaching methods, that favors the protagonist and involvement of the student taking advantage of the benefits of Information Technology and Communications, and the preferences they have for their use. This paper takes into account the possibilities of teaching method based Learning Problems, as well as, other methodological proposals that promote group work and on-line communication between students, using a Moodle 2.0 environment with group work tools and OpenMeetings.
\end{abstract}




\section{INTRODUCCIÓN}

Los estilos de aprendizaje hacen parte integral del ser humano y ello exige unos estilos de enseñanza que los beneficien. Son múltiples los rasgos que precisan la manera en que cada persona aprende, lo que exige especificar el modo en que las personas acceden al conocimiento con el fin de propiciar un proceso de enseñanza que atienda los aspectos cognitivos, emociónales, fisiológicos y contextuales de los individuos (Lozano-Rodríguez, 2008). Es justo aclarar que una buena porción de investigaciones dirigidas a grupos universitarios, se han decantado por el modelo planteado por Kolb (1976), pues se adapta mejor a este tipo de escenarios y de individuos. Una razón fundamental es porque la educación superior centra los procesos de enseñanza en metodologías que fortalecen la autonomía usando la experiencia acumulada por los estudiantes. De igual manera, la teoría de Kolb prioriza la experiencia como un aspecto necesario para alcanzar el aprendizaje. En esta perspectiva, los escenarios de educación superior instalan sus didácticas en la solución de problemas, la observación, la reflexión y la puesta en práctica de los conceptos, entre otras estrategias, que priorizan la experiencia como una manera de alcanzar el aprendizaje. El Aprendizaje Basado en Problemas (ABP) refleja el modo de aprender de las personas en el mundo real al tratar de resolver los problemas que les plantea la vida con los recursos que tengan a su alcance. El estudiante aprende las destrezas para buscar el conocimiento que necesite cuando se presente la ocasión.

La carrera de Ingeniería Informática tiene como objetivo que los estudiantes puedan elaborar sistemas informáticos de aplicación para la gestión y toma de decisiones en organizaciones productivas y de servicio, dentro de sus disciplinas se encuentra la de Inteligencia Artificial la cual se centra en el estudio de modelos y métodos de representación y solución de problemas empleando técnicas de Inteligencia Artificial siendo una de sus principales asignatura, la asignatura de Inteligencia Artificial (IA) que permitirá a los estudiantes diseñar e implementar sistemas que utilicen técnicas de I A para el control del funcionamiento y la toma de decisiones en el sector empresarial e industrial. En la asignatura se abordan temas complejos que requieren del pensamiento abstracto del estudiante lo cual ha tradicionalmente ha generado falta de motivación de los estudiantes, de conocimientos previos, poco aprovechamiento de las clases, tanto durante el curso como en asignaturas posteriores. Con respecto a la bibliografía los libros que se tienen en la asignatura, son meros recursos de consultas, en los mismos resulta difícil interpretar la información que contienen, mucho de ellos tienen aplicaciones que son tan sofisticadas que casi nunca son entendidas por los lectores, o no reúnen las características mínimas de la didáctica con lo cual se pierde totalmente el interés por parte de los lectores, porque simplemente son difícilmente aplicables a realidades cercanas.

Los estudiantes presentan un bajo nivel de autonomía en la búsqueda de la información y elaboración de materiales de estudio, tienen escaso hábitos de utilización de Internet en el autoaprendizaje y poco nivel de síntesis y de habilidades para integrar la información procedente de diferentes fuentes, un elevado nivel de dependencia para con el profesor y fuerte hábito o tendencia persistente a utilizar el aprendizaje memorístico sin pasar por la comprensión de la información almacenada. El estudiante requiere de nuevas formas de enseñanza en el cual las TIC sean incluidas como parte fundamental para el desarrollo de las actividades de aprendizaje, una opción para fortalecer, enriquecer y desarrollar el proceso de enseñanza aprendizaje es vincular el método de ABP con el apoyo de las TIC. En este trabajo 
se presenta una propuesta metodológica para el desarrollo del proceso de enseñanza aprendizaje en la asignatura Inteligencia Artificial con el método de Aprendizaje Basado en Problemas utilizando la plataforma Moodle.

\section{DESARROLLO}

\subsection{Aprendizaje Basado en Problemas y el trabajo en equipo.}

El Aprendizaje Basado en Problemas es un método de enseñanza-aprendizaje centrado en el estudiante en el que éste adquiere conocimientos, habilidades y actitudes a través de situaciones de la vida real. Su finalidad es formar estudiantes capaces de analizar y enfrentarse a los problemas de la misma manera en que lo hará durante su actividad profesional, es decir, valorando e integrando el saber que los conducirá a la adquisición de competencias profesionales. El Aprendizaje basado en Problemas (ABP) se sustenta sobre el principio de utilizar los problemas como punto de partida para la adquisición e integración de nuevos conocimientos (Barrows, 1986).

El desarrollo de este método requiere: compromiso del alumno en su aprendizaje, formación de pequeños grupos, el papel de guía y consultor del aprendizaje del docente, la organización del currículo sobre los problemas que estimulan el aprendizaje y que desarrollan las habilidades en la resolución de problemas (Barrows, 1996).

Recoge muchos elementos pedagógicos que han demostrado ser útiles para mejorar los procesos de enseñanza/aprendizaje: aprendizaje cooperativo y activo, retroalimentación rápida, resolución de problemas del mundo real, etc. Algunas características de esta estrategia son (Barrett, 2005):

Utiliza una metodología de trabajo que desarrolla habilidades cognitivas de alto nivel (resolución de problemas, análisis, toma de decisiones, pensamiento crítico, ...)

Promueve la metacognición y el aprendizaje autorregulado.

Sitúa a los estudiantes en un papel de profesionales activos que intentan resolver un problema o situación próxima a la realidad.

$\square$ Requiere de conocimientos integrados e interdisciplinarios, forma al estudiante para la vida real y genera responsabilidad para asumir sus retos.

Diseñar un currículum basado en el ABP comporta redefinir nuestra concepción de currículum, de tal manera que en éste se ha de considerar: la selección de contenidos prácticos, estructuración basada en conceptos y objetivos de aprendizaje, y la importancia de los procesos que se llevan a cabo. Los estudiantes han de trabajar sobre un problema propuesto en grupos que normalmente son de entre 5 y 8 alumnos. El profesor actúa como tutor que facilita los procesos que se llevarán a cabo y los avances en la línea adecuada para abordar el problema. Su papel incluye, entre otras cosas, escuchar al alumnado haciendo las críticas pertinentes, promover el trabajo en el grupo. 
Formular las preguntas adecuadas para encarrilar bien el trabajo del grupo hacia los objetivos propuestos, orientar en el uso de las fuentes de información, y establecer un buen clima de trabajo entre estudiantes en el grupo. Es decir, el profesor hace de acompañante, guía y orientador, diseña los problemas y ayuda a los alumnos a encontrar, organizar y manejar la información. En la estrategia ABP hay que entender el problema que se plantea es un verdadero desafío que proporciona relevancia y motivación para el aprendizaje. Esto debe ser así, porque con la finalidad de comprender y abordar el problema, los estudiantes han de identificar lo que necesitan aprender (aprendizaje autodirigido).

Además, el problema les conducirá a integrar informaciones procedentes de distintas áreas de conocimiento. De acuerdo con Branda (2009) los objetivos y las tareas que se deben cumplir en el ABP son:

- Utilizar estrategias de razonamiento para proponer hipótesis explicativas.

- Identificar necesidades de aprendizaje.

- Capacitar para trasladar los aprendizajes conseguidos hacia otros problemas.

Tras la presentación del problema, se tiene que hacer ver a los estudiantes la necesidad de un plan de acción para resolverlo. Esto es, se han de proponer una serie de pasos a seguir, uno de los cuales ha de ser ineludiblemente aprender/investigar/estudiar determinadas cosas (objetivos de aprendizaje) y que éstas pueden ser distribuidas entre los miembros del grupo. Basándonos en Moust y colaboradores (2004), ofrecemos, a título orientativo, un ciclo de siete pasos a seguir para apoyar las estrategias ABP:

1. Clarificar términos y conceptos desconocidos del enunciado del problema

2. Definir de manera precisa la situación problemática planteada

3. Analizar el problema: generar una brainstorm o lluvia de ideas de explicaciones y/o hipótesis. En ocasiones puede ser necesario en este punto buscar información.

4. Criticar y/o contrastar las hipótesis y/o explicaciones propuestas y proponer una descripción coherente de todos los procesos, mecanismos o variables que intervienen en el problema.

5. Formular los objetivos de aprendizaje (aprendizaje autodirigido).

6. Rellenar las lagunas de conocimiento mediante el estudio y la búsqueda de información adecuada.

7. Compartir los conocimientos adquiridos con los compañeros del grupo, evaluar la idoneidad de conocimientos y planteamientos para la resolución del problema; y si no fueran idóneos, repetir el ciclo a partir de algún paso determinado.

Tras llegar a una solución del problema, los estudiantes y el profesor han de conocer lo que se ha aprendido, y esto puede lograrse a través de la evaluación. En la evaluación se debería tener en cuenta (Egido et al., 2007): los conceptos y procedimientos; las estrategias empleadas, asumiendo que el estudiante ha debido ser un participante activo en el grupo y, a su vez, autónomo; y el trabajo en equipo. 
Por tanto, se ha de evaluar la aportación individual (el trabajo generado para la solución del problema), la aportación del grupo (trabajo producido por el grupo), a los compañeros del grupo (coevaluación o evaluación de un alumno a sus compañeros) y el estudiante a sí mismo (autoevaluación) (Morales y Landa, 2004). Asimismo, no se puede olvidar la evaluación a realizar por el profesor, que puede ser diagnóstica (para obtener información sobre conocimientos previos, o de determinados conocimientos o tareas), formativa (para averiguar si se alcanzan los objetivos de aprendizaje) o sumativa (para asignar calificaciones finales a los estudiantes). Es imprescindible, puesto que el ABP incide en el aprendizaje autodirigido, que los estudiantes tienen que saber muy bien desde el principio los criterios de evaluación. Por último, se destacan las características que según Branda (2009) debe cumplir un problema para aplicar la estrategia ABP. Según este autor, ha de ser no estructurado (abierto), presentar la información de manera progresiva, que propicie la discusión del grupo y que incluya frases controvertidas. También es muy recomendable que contenga elementos en los que pueda sentirse vinculado el estudiante y que refleje la realidad del futuro mundo laboral asociado a su titulación. Como es obvio, el problema planteado tendrá como referencia los contenidos académicos que el profesor pretenda cubrir.

\subsection{Roles del Aprendizaje Basado en Problemas}

Profesor: Estimular la discusión y desafiar el pensamiento e ideas de los estudiantes

El Rol del profesor, instructor o docente, es asegurarse que los estudiantes tengan claro el rol del que lo dirige. El rol del profesor es estimular la discusión. Los profesores no son las fuentes de información sobre el problema, aun cuando tengan conocimiento en algunos aspectos del mismo. El profesor no enseña en el sentido convencional de la palabra, pero facilita el proceso de aprendizaje a través de preguntas que apuntan a provocar el pensamiento y la discusión entre los estudiantes. El profesor debe desafiar el pensamiento e ideas de los estudiantes si tiene dudas de que entienden lo que están discutiendo. La pregunta más común de un instructor es “¿Por qué?”.

Estudiantes: Cuestionarse a ellos mismos y no depender del profesor para este desafío, trabajar en grupos pequeños y de manera colaborativa.

Los estudiantes deben cuestionarse a ellos mismos y no depender del profesor para este desafío. Mediante los cuestionamientos entre sí, ellos analizan, diseminan y discuten la información relacionada con el problema y los principios y conceptos que el conocimiento ilustra. Los estudiantes aprenden más efectivamente, desarrollando los atributos y propiedades que son objetivos del aprendizaje basado en problema, en grupos pequeños y auto-dirigido, siempre y cuando el profesor posea habilidades; entre las cuales tenemos, la facilitación del aprendizaje, promover el pensamiento crítico, promover el aprendizaje basado en problemas del grupo, promover el aprendizaje individual.

\subsection{Propuesta de la didáctica de la asignatura para el trabajo grupal y el aprendizaje basado en problema}


La propuesta es la siguiente como forma organizativa incorporar el seminario de solución de problemas y ejercicios para el desarrollo del trabajo independiente, utilizar como medios didácticos el EVEA pero en modalidad grupal además de crear tutoriales interactivos que ayudan a una mejor comprensión del problema a tratar, como forma de evaluación incorporar un trabajo extraclase en equipos de pocos estudiantes deben forman grupos de 35 personas. De esta manera se podrán cumplir los objetivos educativos e instructivos que plantea la asignatura en particular estos dos que se mencionan a continuación adquirir hábitos de trabajo en grupo y desarrollar el espíritu de autosuperación.

En la primera sesión el profesor propone varios problemas que se constituyen en trabajos extraclases, y el grupo escoge uno. Cada problema conlleva unos objetivos de aprendizaje que los alumnos deben descubrir. De cada reunión, tutorada o no, se escribe un acta que recoja el trabajo del grupo. Cada acta es entregada al profesor electrónicamente. Se puede clasificar las mejoras realizadas en tres ámbitos: entregables, tutorías on-line y evaluación.

En la asignatura, para el tema 1, se pretende hacer una aproximación general de cómo solucionar un problema mediante diferentes métodos de búsquedas. Conocer cuál es la estrategia a seguir para construir un sistema que resuelva un problema específico. Se comienza por indicar un planteamiento de definición de un problema; para luego continuar con el estudio de cada uno de los diferentes tipos de búsquedas, sean estas informadas o no.

Como ejemplos de estos problemas tenemos el del viajante de comercio, el 8 puzzle, las 8 reinas, los caníbales y monjes, la torre de Hanói, etc, para ser visto durante el curso; y como trabajo extraclase se orientan problemas más cercanos a la vida real como pueden ser: la planificación de horarios de clase, la configuración de hardware compatible, la planificación de rutas, la planificación de producción, los problemas de asignación de recursos, la evaluación de riesgos en inversiones.

\subsection{Entregables}

Una vez formados los grupos y elegido el proyecto, los alumnos deben presentar los siguientes entregables:

> Acta de reunión: Al finalizar una reunión de grupo (sea ante profesor o no) los alumnos deben reportar un acta refleje la evolución en la discusión y decisiones del proyecto. Dicha acta se entrega por Moodle.

$>$ Talleres: A la tercera semana, con el objetivo de proporcionar ideas y herramientas a los grupos se realiza una sesión de talleres, de dos horas sobre temas de la asignatura. Al finalizar la sesión, cada alumno de entrega de un resumen de lo discutido, y en qué les puede servir en su proyecto.

$>$ Control: En dos semanas concretas (la tercera y la duodécima de un total de 15 semanas) los alumnos deben entregar un control de forma grupal. En este entregable los alumnos especifican a qué se comprometen como trabajo, características de la 
aplicación, funcionalidades, tareas, responsabilidades para cada tarea y una previsión de tiempo para cada tarea. Se trata de una definición clara del proyecto. En la primera entrega se les pide ambición en los objetivos. En el segundo control se les pide realismo, a tres semanas de la presentación oral y entrega del proyecto.

> Co y autoevaluación: Al finalizar la entrega de cada control se pide a los alumnos que de forma individual contesten una encuesta de coevaluación de sus compañeros de grupo y auto-evaluación según una plantilla de preguntas. Se realizará otra actividad de este tipo la última semana de clase con la presentación oral.

> Presentación oral y entrega del proyecto: En la última sesión un alumno del grupo presenta el proyecto en 15 minutos ante el profesor. Al final de la sesión cada grupo lista de mejor a peor los trabajos de los demás grupos presentados en la sesión, sin puntuar. Cada grupo entrega a través de la plataforma Moodle 2.0 la siguiente documentación: El Portafolio del trabajo, en el cual debe estar la memoria escrita del proyecto, la presentación oral, transparencias de la presentación oral y por último la aplicación informática, Programa ejecutable del proyecto.

Al finalizar la entrega de cada control, el profesor sube al moodle para cada grupo un informe de seguimiento del proyecto, que permite a los alumnos saber la valoración del trabajo que realiza el profesor, y qué puntos deben potenciar para la entrega final. Este informe tiene el formato de encuesta según una plantilla de preguntas.

Desde la primera reunión destacamos a los alumnos la importancia de explicar claramente en las actas de las discusiones y acuerdos que realizan, pidiéndoles además este curso que asuman roles de responsabilidad en los diferentes aspectos del proyecto. Cada alumno será responsable de una faceta del proyecto y colaborará en otros aspectos como miembro del grupo.

\subsection{Estrategia metodológica y organizativa}

Para la realización de las actividades, los 22 alumnos integrantes del curso se dividieron en cinco grupos de tamaño variable entre 3 y 5 participantes. Durante las seis semanas que duró el módulo ABP, todas las sesiones de clases práctica se realizaron a modo de taller de trabajo. Debido a la disponibilidad de un aula cuyo mobiliario no estaba anclado al suelo, antes de iniciar la sesión de trabajo, los alumnos redistribuían mesas y sillas con el fin de formar una mesa grande para cada grupo de trabajo.

Las sesiones en el aula, de hora y media de duración, se dedicaron a la presentación de las actividades, a la aclaración de dudas sobre el procedimiento y los contenidos del trabajo y a la explicación, previa a la realización de la actividad, de conceptos cuya complejidad podría haber constituido un escollo. Una vez que todos los equipos habían comprendido los objetivos de la actividad, se fijaba una fecha para su entrega. El trabajo -uno por grupo- debe ser entregado a través de la plataforma Moodle de la asignatura. Se habilitó para ello un espacio Wiki en dicha plataforma. 
Mucha de la información que los alumnos podían consultar para abordar la resolución del problema se les proporcionó desde el principio, evitando de este modo que tuvieran que encargarse ellos de la búsqueda o selección de materiales. Fue necesaria la utilización de las TIC (Tecnologías de la Información y la Comunicación) como fuerte apoyo para impulsar la colaboración y la cohesión de grupo, así como para facilitar las tareas de tutorización, retroalimentación y evaluación y, por último, para realizar un control del grado de seguimiento por parte de los alumnos. La plataforma virtual de la asignatura contó con un foro de dudas, comentarios y aportaciones. Se concibió como una herramienta esencial para el seguimiento y control del proceso de aprendizaje y, dentro de éste, del nivel de implicación y del interés de los alumnos. Mediante este foro, único para el conjunto de la actividad ABP, se pretendía impulsar la colaboración, no ya entre subgrupos, sino entre todos los alumnos del curso. Mediante el foro se pretendía, además, obtener un reflejo de la dinámica de trabajo, de las dudas que surgieran y del nivel de colaboración que se establecía entre los alumnos.

En relación al sistema de trabajo, la secuencia organizativa habitual fue la siguiente:

1. Presentación de la actividad.

2. Lectura y análisis de materiales.

3. Presentación colectiva de dudas y dificultades para cumplimentar la actividad.

4. Elaboración de la actividad.

5. Subida de materiales a la plataforma Moodle.

6. Corrección por parte del profesor.

7. Retroalimentación colectiva: análisis en el aula de errores, carencias, etc.

\subsection{Tutorías on-line}

Se definen dos modalidades como forma de utilización de la herramienta Open Meetings para realizar las tutorías on-line:

Tutorías virtuales: En horas de consulta presenciales el profesor se conecta a Open Meetings en la que se define como moderador. No se graban las sesiones. Usualmente son dos (profesor y alumno) los usuarios conectados.

Reuniones grupales: Se abre una actividad Open Meetings para cada grupo de trabajo de alumnos, donde el moderador es el primero que se conecta y hacen una reunión de trabajo. El grupo de trabajo tiene definida una actividad Open Meetings concreta del resto de grupos. La actividad dispone de una pizarra propia para cargar documentos, que permanece entre sesiones si nadie la borra. Las sesiones se pueden grabar, y el profesor tiene acceso a las grabaciones hechas por el grupo.

\subsection{Evaluación del aprendizaje}

Se realizó una evaluación continua, a través de distintos procedimientos: 
a) Evaluación de cada una de las actividades realizadas mediante análisis y valoración de los resultados previamente incorporados a la Wiki.

b) Evaluación mediante tutorías de la aportación individual al trabajo de grupo.

c) Evaluación de la participación individual en el foro.

d) Evaluación del trabajo extra clase.

\subsection{Valoraciones de la aplicación de la propuesta.}

Las encuestas realizadas a los estudiantes indican que su motivación y su participación activa en clase han aumentado. Además, los resultados académicos apuntan también a una mejora cualitativa de la adquisición de habilidades durante el curso. El uso de herramientas on-line permitió mantener un contacto más directo con los alumnos fuera del aula, facilitando a los alumnos que se puedan reunir y realizar el proyecto si están en lugares distintos.

Las actividades dirigidas, como los talleres o los entregables representan trabajo adicional, que ayudan al alumno a organizar la complejidad del proyecto. Los alumnos manifiestan dificultades en el redactado de las actas, y en expresarlas ideas que se discuten en las reuniones. La competencia de trabajo en equipo suele estar evaluada por el resultado del trabajo del equipo, bien por su resultado final (el propio trabajo) o bien por resultados parciales, como pueden ser la elaboración de una normativa, el mapa de responsabilidades o un cronograma. Se ha demostrado que ante resultados parciales o finales, evaluados de forma común no suele haber correspondencia con la adquisición de la competencia por los individuos que configuran el grupo.

Por tanto, la evaluación de las evidencias que deja cada miembro del equipo es la forma de evaluar la adquisición de competencias; esta labor necesita el apoyo de los sistemas de Learning Analytics, que facilitan la visualización de los datos y actúan a modo de alarmas tanto para evaluar como para favorecer el aprendizaje de la competencia del individuo. La Evaluación del trabajo en grupo no solo por el resultado final sino por el desempeño durante el trabajo.

\section{CONCLUSIÓN}

El ABP es un método que se orienta a la solución de problemas que son seleccionados o diseñados para lograr el aprendizaje de ciertos objetivos de conocimiento, estimula el trabajo colaborativo y se favorece con el empleo de las TIC.

El trabajo en equipo es una metodología que a los estudiantes les resulta muy enriquecedor, desarrolla su capacidad reflexiva, les permite argumentar, investigar, comunicarse entre sí, experimentar, crear, salirse de los parámetros y al debatir los puntos pueden percatarse que ellos tienen que decir y al decir aprenden, no solo los conceptos, sino también habilidades propias del desarrollo académico y profesional sobre temas clave para el progreso de su habilidad en la lectura y la escritura. 


\section{BIBLIOGRAFÍA}

1. Barrows, H. S. 1986. A Taxonomy of problem-based learning methods, Medical Education, no 20, pp. 481-486.

2. Barrows, H. S. 1996. Problem-Based Learning in medicine and beyond: A brief overview, en Wilkerson, L. y Gijselaers, W. H. (eds.): Bringing Problem-Based Learning to Higher Education: Theory and Practice, Jossey-Bass Pub., San Francisco, pp. 3-12.

3. Branda, L. A. (2009). El aprendizaje basado en problemas. De herejía artificial a res popularis. Educación Médica, 12 (1), 11-23.

4. Cabero, J. y Román, P. (Coord.) (2006). E-Actividades. Un referente básico para la formación en Internet. Sevilla: Editorial MAD.

5. Egido, I., Aranda, R., Cerrillo, R., De la Herrán, A., De Miguel, S., Gómez, M., Hernández, R., Izuzquiza, D., Murillo, F. J., \& Pérez, M. (2006). Aprendizaje basado en problemas: Estrategia metodológica y organizativa del currículum para la calidad de la enseñanza en los estudios de Magisterio. Revista Interuniversitaria de Formación del Profesorado, 20(3), 137-149.

6. Egido, I., Aranda, R., Cerrillo, R., De la Herrán, A., De Miguel, S., Gómez,M., Hernández, R., Izuzquiza, D., Murillo, F. J., Pérez, M. \& Rodríguez,R. (2007). El aprendizaje basado en problemas como innovación docente en la universidad: Posibilidades y limitaciones. Educación y Futuro, 16,85-100.

7. Gil Flores, J., Álvarez Rojo, V., García Jiménez, E. y Romero Rodríguez, S. (2004). La Enseñanza Universitaria. Planificación y Desarrollo de la Docencia. Madrid: EOS Universitaria.

8. Kolb. (1976). Learning Style Inventory, Self-Scoring Test and Interpretation Booklet. Boston: McBer and Company.

9. Lozano Rodríguez, A. (2008). Estilos de aprendizaje y enseñanza: un panorama de la estilística educativa. Distrito Federal, México: Trillas.

10. Llorens-Molina, J. A. (2010). El aprendizaje basado en problemas una estrategia para el cambio metodológico en los trabajos de laboratorio. Química Nova, 994-999.

11. Molina, J. A., García, A., Pedraz, A. \& Antón, M. A. (2003). Aprendizaje basado en problemas: una alternativa al método tradicional. Revista de la Red Estatal de Docencia Universitaria, 3 (2), 79-85.

12. Morales, P. \& Landa, V. (2004). Aprendizaje basado en problemas. Theoria, 13, 145157.

13. Mossuto, M. (2009). Problem-based learning: Student engagement, learning and contextualised problem-solving. Adelaide: NCVER. www.ncver.edu. au/publications/2198.html.

14. Moust, J. H. C., Bouhuijs, P. A. J. \& Schmidt, H. G. (2004). El aprendizaje basado en problemas: Guía del estudiante. Cuenca: Ediciones de la UCLM.

15. Prieto, A., Barbarroja, J., Reyes, El., Montserrat, J., Díaz, D., Villaroel, M. \& ÁlvarezMon, M. (2006). Aula Abierta, 87, 171-194 